\title{
Impact of higher-order optical fiber modes for photon-triplet generation
}

\author{
K. Garay-Palmett ${ }^{a, *}$ and F. Domínguez-Serna ${ }^{b}$ \\ ${ }^{a}$ Departamento de Óptica, \\ Centro de Investigación Científica y de Educación Superior de Ensenada, \\ B.C., 22860, México. \\ *e-mail:kgaray@cicese.edu.mx \\ ${ }^{b}$ Cátedras Conacyt, Centro de Investigación Científica y de Educación Superior de Ensenada, \\ B.C., 22860, México.
}

Received 11 December 2020; accepted 29 December 2020

\begin{abstract}
This paper presents a study of the phasematching properties of the third-order parametric down-conversion process in multimode optical fibers with a step-index profile. Through this process, entangled photon triplets can be generated in the spontaneous regime. We show that phasematching is achieved in a wide range of fiber geometries, provided that the pump field propagates in a higher-order mode. Furthermore, we show the nonlinear coefficient and the absolute emitted flux for each source configuration, evaluated from expressions derived by us in previous theoretical studies concerning the spontaneous and stimulated emission regimes.
\end{abstract}

Keywords: Quantum optics; nonlinear optics; optical fibers; three-photon states.

PACS: 42.50.-p; 42.65.-k; 42.65.Lm

\section{Introduction}

For implementing different protocols in quantum information processing, an essential need is the availability of multipartite states. Currently, one of the biggest challenges in quantum optics is to generate entangled multi-photon systems. Since the first demonstration of spontaneous parametric down-conversion (SPDC) in a second-order nonlinear medium [1], photon-pair entanglement has been used for validation of quantum mechanics [2] and exploited to the development of quantum technologies [3]. Photon pairs can also be generated in optical fibers by the process of spontaneous four-wave mixing (SFWM) [4]. In both cases, the process can be engineered to generate two-photon states with particular entanglement properties [5,6].

A step forward in developing non-classical light sources is the generation of three-photon states, for which have been proposed and implemented various schemes based on cascaded second-order processes [7]. More recently, there has been a growing interest in the study and design of alternatives for generating three-photon states in media with third-order nonlinearity, mainly in guided devices [8-14]. However, at present, experimental demonstrations remain a challenge to overcome [15].

It was previously proposed to generate photon triplets in optical fibers guided by air, based on the third-order spontaneous parametric down-conversion process (TOSPDC) [8]. In this process, a pump photon with frequency $\omega_{p}$ decays spontaneously in a photon triplet with frequencies $\omega_{r}, \omega_{s}$, and $\omega_{i}$. As a parametric interaction process, the conditions of energy and momentum conservation must be fulfilled. In that work, we show that if the pump field is allowed to travel in the first excited fiber mode while generated wave-packets propagate in the fundamental mode, phasematching is achieved for subwavelength fibers (a few hundred nanometers). This scheme allows the emission of frequency non-degenerate and degenerate photon triplets. Shortly after, a similar technique was proposed using more conventional fibers and considering that the pump propagates in the $L P_{03}$ mode [16]. More recently, we also have studied the process of third-order parametric downconversion (TOPDC), in which, additionally to the pump, a seed field is launched to the fiber that overlaps at least one generation spectral mode so that the emission becomes stimulated [14]. This paper presents an analysis of the phasematching properties for TOSPDC (and TOPDC) in multimode fibers with a step-index profile. In contrast to our previous studies, here we let the pump field propagate in a spatial mode, which can be any of the higher-order modes supported by the optical fiber, belonging to the kind of linearly polarized modes $L P_{0 m}$ with $m=1,2,3, \cdots$. Under these conditions, phase-matching occurs in fibers with larger core radii, allowing longer fiber lengths and higher pump powers, leading to higher emission rates [17].

This paper also shows the nonlinear coefficient associated with the four-field interaction and the emitted flux obtained for each source geometry concerning the spontaneous (TOSPDC) and stimulated emission regimes (TOPDC). As described below, although in general the obtained nonlinear coefficient is low, the fact that the phasematching occurs for fibers with core radii greater than those assumed in [8] allows us to consider longer fiber lengths. A relevant issue to be taken into account is that our technique's successful implementations rely on the viability of launching into the fiber the greater available pump power in the corresponding higher-order mode. In this regard, several methods have been proposed through which it is possible to excite selectively and controllably a particular higher-order mode in an optical fiber [18-21]. On the other hand, as we have emphasized, the 
TOSPDC emitted flux is proportional to the nonlinear coefficient's square. Hence, the use of fibers made from highly nonlinear materials $[22,23]$ constitutes a reasonable alternative to implement photon-triplet sources with higher emission rates.

\section{Theory of third-order parametric down- conversion}

\subsection{Spontaneous regime}

We have previously carried out a comprehensive theoretical study of TOSPDC in optical fibers, comprising the pulsed and monochromatic pump regimes [8,17]. Assuming that all four fields are linearly polarized along the $x$-axis and propagate in the same direction along with the fiber ( $z$-axis), and following a standard perturbative approach, we showed that the state produced by third-order spontaneous parametric downconversion is $|\Psi\rangle=|0\rangle_{r}|0\rangle_{s}|0\rangle_{i}+\eta\left|\Psi_{3}\right\rangle$, written in terms of the three-photon component of the state $\left|\Psi_{3}\right\rangle$

$$
\begin{aligned}
\left|\Psi_{3}\right\rangle & =\int d \omega_{r} \int d \omega_{s} \int d \omega_{i} \\
& \times f\left(\omega_{r}, \omega_{s}, \omega_{i}\right)\left|\omega_{r}\right\rangle_{r}\left|\omega_{s}\right\rangle_{s}\left|\omega_{i}\right\rangle_{i}
\end{aligned}
$$

where $\eta$ is a constant related to the conversion efficiency. The function $f\left(\omega_{r}, \omega_{s}, \omega_{i}\right)$ is the joint spectral amplitude, which describes the spectral correlation properties of the three-photon state, and is given in terms of the pump spectral amplitude (PSA) $\alpha(\omega)$, and the phasematching function (PM) $\phi\left(\omega_{r}, \omega_{s}, \omega_{i}\right)$

$$
f\left(\omega_{r}, \omega_{s}, \omega_{i}\right)=\alpha\left(\omega_{r}+\omega_{s}+\omega_{i}\right) \phi\left(\omega_{r}, \omega_{s}, \omega_{i}\right),
$$

with

$$
\begin{aligned}
\phi\left(\omega_{r}, \omega_{s}, \omega_{i}\right) & =\operatorname{sinc}\left[L \Delta k\left(\omega_{r}, \omega_{s}, \omega_{i}\right) / 2\right] \\
& \times \exp \left[i L \Delta k\left(\omega_{r}, \omega_{s}, \omega_{i}\right) / 2\right]
\end{aligned}
$$

written in turn in terms of the fiber length $L$ and the phase mismatch $\Delta k\left(\omega_{r}, \omega_{s}, \omega_{i}\right)$

$$
\begin{aligned}
\Delta k\left(\omega_{r}, \omega_{s}, \omega_{i}\right) & =k_{p}\left(\omega_{r}+\omega_{s}+\omega_{i}\right)-k_{r}\left(\omega_{r}\right) \\
& -k_{s}\left(\omega_{s}\right)-k_{i}\left(\omega_{i}\right)+\Phi_{N L}
\end{aligned}
$$

where $\Phi_{N L}$ is a non-linear contribution derived from selfphase and cross-phase modulation processes, which is proportional to the pump power $[17,24]$. Note that the energy conservation constraint is apparent in the argument of the first term of the phase mismatch [see Eq. (4)]. For the pulsed pump configuration, we assume that the pump has a Gaussian spectral envelope $\alpha\left(\omega_{p}\right)=\exp \left[-\left(\omega_{p}-\omega_{p 0}\right)^{2} / \sigma^{2}\right]$, with $\omega_{p 0}$ and $\sigma$ the central frequency and bandwidth, respectively. Plots of the joint spectral intensity (JSI), defined as $\left|f\left(\omega_{r}, \omega_{s}, \omega_{i}\right)\right|^{2}$, have been shown in Refs. [8, 17]. Essentially, the JSI is like a "membrane" of narrow width along the direction $\omega_{s}+\omega_{i}+\omega_{r}$, and much larger widths along with the two perpendicular directions, a feature that reveals the existence of spectral correlation in the three-photon state.

For practical implementations of photon-triplet sources, results essential to know the dependence of the photon emission rate in terms of all experimental parameters. We have derived an expression to calculate the brightness of photontriplet sources based on TOSPDC, defined as the number of single photons detected in one of the three-generation modes (e.g. the $r$ mode) per unit time, which under ideal detection efficiency conditions, also corresponds to the number of photon triplets emitted per unit time. For the state given in Eq. (1), and assuming a pulsed pump, the TOSPDC emitted flux can be calculated as $N_{s}=\sum_{k_{r}}\left\langle\Psi_{3}\left|\hat{a}^{\dagger}\left(k_{r}\right) \hat{a}\left(k_{r}\right)\right| \Psi_{3}\right\rangle R$, with $R$ the pump repetition rate. After some algebra can be demonstrated that $N_{s}$ is explicitly given by $[8,17]$

$$
\begin{aligned}
N_{s} & =\frac{3(2)^{5 / 2} \hbar c^{3} n_{p}^{3}}{\pi^{5 / 2} \omega_{p 0}^{2}} \frac{L^{2} \gamma^{2} p}{\sigma} \int d \omega_{r} \int d \omega_{s} \int d \omega_{i} \\
& \times \frac{k_{r}^{\prime} \omega_{r}}{n_{r}^{2}} \frac{k_{s}^{\prime} \omega_{s}}{n_{s}^{2}} \frac{k_{i}^{\prime} \omega_{i}}{n_{i}^{2}}\left|f\left(\omega_{r}, \omega_{s}, \omega_{i}\right)\right|^{2},
\end{aligned}
$$

where $\hbar$ is the Planck's constant, $c$ the speed of light in vacuum, $n_{p}$ the refractive index evaluated at the pump central frequency, $p$ and $\sigma$ are the pump average power and bandwidth, respectively, and $\gamma$ is the nonlinear coefficient that governs the TOSPDC process, given by

$$
\gamma=\frac{3 \chi^{(3)} \omega_{p 0}}{4 \epsilon_{0} c^{2} n_{p}^{2} A_{e f f}}
$$

where $A_{\text {eff }}$ is the effective interaction area among the four fields [9]. In Eq. (5), $k^{\prime} \equiv k^{\prime}(\omega)$ represents the first frequency derivative of $k(\omega)$. An expression of the TOSPDC emitted flux in the monochromatic pump regime can be obtained by taking the limit $\sigma \rightarrow 0$ in Eq. (5).

In the general case, is not trivial to solve analytically the triple frequency integral in Eq. (5). However, a numerical analysis of the emitted flux, as given by Eq. (5), for specific source designs reveals that the emission rate of photon triplets has a linear dependence on the fiber length and pump power, while is constant for the pump bandwidth (maintaining the pulse energy constant) within the phasematching bandwidth [17]. This behavior is similar to that observed in SPDC. A critical issue to consider is that $N_{s}$ varies with the square of the parameter $\gamma$, which in turn is proportional to the nonlinear refractive index of the fiber material and inversely proportional to the effective area [see Eq. (6)]. Thus, it is desirable to maximize the spatial overlap between the four fields involved in increasing the emitted flux and consider optical fiber manufactured with materials exhibiting high third-order electrical susceptibility values. 


\subsection{Stimulated emission}

Stimulated emission tomography (SET) is a technique that uses a seed input field in addition to the pump, which overlaps at least one of the output modes, such that the emission becomes stimulated. SET was initially proposed and experimentally implemented to study the generation of photonpairs [25-27] and has also been studied in the third-order nonlinear regime [10-14]. The input seed field is taken as a coherent state of the form $\hat{D}\left(\left\{\beta_{i}\right\}\right)|v a c\rangle$ with an initial average photon number $\left|\beta_{0}\right|^{2}$, that evolves by the coupled nature of the fields into $\hat{D}\left(\left\{\bar{\beta}_{i}(t)\right\}\right) \mid$ vac $\rangle$, while the pump field coherent state $\hat{D}\left(\left\{\alpha_{p}\right\}\right)|\mathrm{vac}\rangle$ is assumed constant. For TOPDC in the undepleted pump approximation, the output state can be written as follows [14]:

$$
\begin{aligned}
\left|\psi_{\text {out }}\right\rangle & =\hat{D}\left(\left\{\alpha_{p}\right\}\right) \hat{D}\left(\left\{\bar{\beta}_{i}(t)\right\}\right) \\
& \left.\times \mathscr{N}(\mid \text { vac }\rangle+\eta_{I I I}\left|\Psi_{3}\right\rangle+\eta_{I I}\left|\Psi_{2}\right\rangle\right),
\end{aligned}
$$

where $\hat{D}\left(\left\{\alpha_{p}\right\}\right)$ and $\hat{D}\left(\left\{\bar{\beta}_{i}(t)\right\}\right)$ represent the pump and seed displacement operator at the output of the medium. The notation $\eta_{I I I}$ and $\eta_{I I}$ were used to emphasize their dependency with the generation of the normalized triplet states $\left|\Psi_{3}\right\rangle$ and photon-pair like states $\left|\Psi_{2}\right\rangle$, where the former was defined in Eq. (1), and the latter can be written as follows

$$
\begin{aligned}
\left|\Psi_{2}\right\rangle & =\sqrt{3} \frac{\eta_{I I I}}{\eta_{I I}} \int d \omega_{r} \int d \omega_{s} \int d \omega_{i} \\
& \times f\left(\omega_{r}, \omega_{s}, \omega_{i}\right) \beta^{*}\left(\omega_{i}\right)\left|\omega_{r}\right\rangle\left|\omega_{s}\right\rangle .
\end{aligned}
$$

It is possible to calculate the average number of photons under the above conditions, simply as

$$
\begin{aligned}
N & =\int d \omega_{r}\left\langle\psi_{\text {out }}\left|\hat{a}^{\dagger}\left(\omega_{r}\right) \hat{a}\left(\omega_{r}\right)\right| \psi_{\text {out }}\right\rangle \\
& =N_{0}+N_{I}+N_{I I},
\end{aligned}
$$

where the three terms in (9) are obtained by substitution of (7) in (9). Those terms account for the following: $N_{0}$ is the spontaneous number of photons as $3\left|\eta_{I I I}\right|^{2}$, while $N_{I}$ and $N_{I I}$ are the stimulated throughput of photons, which come from the seed overlapping one or two output modes at the same time. It can be shown that the stimulated throughput is of the form

$$
\begin{aligned}
N_{I} & =2 N_{0}\left|\beta_{0}\right|^{2} \int d \omega_{r} \int d \omega_{s} \\
& \times\left|\int d \omega_{i} f\left(\omega_{r}, \omega_{s}, \omega_{i}\right) \tilde{\beta}^{*}\left(\omega_{3}\right)\right|^{2} \\
N_{I I} & =\frac{N_{0}}{2}\left|\beta_{0}\right|^{4} \int d \omega_{r} \\
& \times\left|\int d \omega_{s} \int d \omega_{i} f\left(\omega_{r}, \omega_{s}, \omega_{i}\right) \tilde{\beta}^{*}\left(\omega_{s}\right) \tilde{\beta}^{*}\left(\omega_{i}\right)\right|^{2},
\end{aligned}
$$

where $\beta(\omega)$ is the spectral envelope of the seed field, and was written as $\beta(\omega)=\beta_{0} \tilde{\beta}(\omega)$, such that $\int d \omega|\tilde{\beta}(\omega)|^{2}=1$.

\section{Exploration of fiber geometries}

\subsection{Phase-matching techniques for TOSPDC}

The TOPDC processes are conditioned to the fulfillment of the phase-matching constraint $\Delta k=0$ [see Eq. (4)]. It has been emphasized that due to the wide spectral separation between the pump frequency and frequencies in which photon triplets are expected to be emitted, it is not simple to get phasematching for cases in which the four interacting fields propagate in the same transverse fiber mode. As an alternative, we previously proposed a multimode TOSPDC scheme in thin optical fibers guided by air. We assumed that the three generated wavepackets propagate in the fundamental mode of the fiber $\left(\mathrm{HE}_{11}\right)$. While the pump field propagates in the excited mode $\mathrm{HE}_{12}$, as considered for third-harmonic generation in reference [28]. Focusing on the degenerate emission case, i.e., when the three photons in a triplet are emitted at the same frequency, we found that for a particular emission frequency, there is a core radius for which phase matching occurs [8], (henceforth we will refer to this as the phasematching radius $r_{d e g}$ ). We also showed that the proposed scheme allows the generation of frequency non-degenerate photon triplets [17].

The phasematching proposal assumes that the pump field propagates in the $\mathrm{HE}_{12}$ mode demands subwavelength dimension optical fibers. For example, to generate photon triplets in the telecommunications band or higher frequencies, the phasematching radius must be less than $0.4 \mu \mathrm{m}$ [8]. Ultra-thin fibers have the advantage of providing high values of the nonlinear coefficient $\gamma$ [see See Eq. (6)] if most of the light is highly confined into the core [29], which also could lead to higher emission rates. However, although these fibers can be made through the current tapers technologies, the lengths for the core radius remains constant are restricted to a few centimeters. For example, recent experimental work shows that it is possible to obtain a uniform core radio of $\sim 445 \mathrm{~nm}$ along a length of $9 \mathrm{~cm} \mathrm{[30]}$. Short interaction lengths represent a disadvantage for fiber-based TOSPDC sources' practical implementations since the emitted flux is in general proportional to the fiber length. On the other hand, the pump power that can be coupled into subwavelength optical fibers is limited to the fiber damage threshold. As an alternative, Ref. [16] proposed a scheme to generate photon triplets in more conventional optical fibers (larger core radius and lower dielectric contrasts), assuming a configuration in which the pump field propagates in the $\mathrm{LP}_{03}$ fiber mode, and generated photons propagate in the fundamental mode.

Current technology allows great flexibility in designing optical fibers with different dispersion profiles, enabling the fabrication of step-index fiber (SIF), gradient index fibers (GIF), and photonic crystal fibers (PCF), which can be singlemode or multimode at the wavelength of interest. Motivated by the wide range of fiber geometries available, we have analyzed the TOPDC phasematching properties in multimode 
SIF, restricting our treatment to linearly polarized modes (LP) since we are focusing on more conventional fibers for which the index contrast is low. In the analysis, the core radius $r$ and the dielectric contrast $\Delta_{n}=\left(n_{1}-n_{2}\right) / n_{1}$ (with $n_{1}$ and $n_{2}$, the refractive index of the core and cladding material, respectively) have been set as free parameters because, in general terms, these control the fiber dispersion properties.

For a particular fiber, the number of transverse modes in which coupled light can travel is determined by the optical frequency. The fundamental mode $\mathrm{LP}_{01}$ is characterized by the highest effective refractive index, while higher modes experience refractive indices whose value decreases as the optical mode order increases. Thus, the uppermost confined mode supported by a fiber propagates with a refractive index close to the cladding's refractive index. This behavior can be observed in Fig. 1a) where we show the effective refractive index of the first ten modes, belonging to the class of modes $L P_{0 m}(m=1,2,3, \cdots)$, supported by a SIF characterized by $r=10 \mu \mathrm{m}$ and $\Delta_{n}=0.02$, for light at $0.532 \mu \mathrm{m}$. Each of these modes has a particular spatial intensity profile, as can be seen in Figs. 1b)- e), whose radial distribution is determined by the $m$ parameter. In this case, all modes exhibit azimuthal symmetry.

We have explored the TOPDC phasematching properties to generate degenerate photon triplets at $1.596 \mu \mathrm{m}$ in fibers with $\Delta_{n}=0.02-0.06$. For this, we assume that emitted photons propagate in the fundamental mode, while the pump field, centered at $0.532 \mu \mathrm{m}$, propagates in a higherorder mode. From our analysis, we have found that for a fixed $\Delta_{n}$ a set of core radii, the condition $\Delta k=0$ is fulfilled. Each core radius is linked to a pump field propagating in a spe- cific higher-order mode, following a monotonically increasing relation. The core radius value increases as the transverse mode order increases. This result is shown in Fig. 2a). The phasematching radius has been plotted as a function of the mode order and the fiber dielectric contrast. In this figure, we can see that for the considered $\Delta_{n}$ range phasematching is achieved in fibers with core radius between 2 and $10 \mu \mathrm{m}$ approximately, which are fiber with conventional dimensions, and for $L P_{0 m}$ modes with $m \geq 3$. Larger values of $\Delta_{n}$ lead to phasematching for lower order modes and at small core radii. In the case of $\Delta_{n}=0.02$, for example, phasematching occurs only from the $\mathrm{LP}_{04}$ mode. Note that phasematching with a pump propagating in the $L P_{02}$ mode becomes possible in fibers with much higher dielectric contrast, such as PCFs or nanofibers, in which light is guided by air, see [8].

In Ref. [17] was showed that non-degenerate TOSPDC is also possible under the scheme proposed in Ref. [8]. Here we extend that analysis to include cases in which the pump field is allowed to propagate in fiber modes of higher orders than that considered in those works. In Fig. 3, panels a) and c), we plot the phase-matched frequencies $\omega_{r}$ and $\omega_{i}$ as a function of the pump frequency $\omega_{p}$ for different values of the dielectric contrast ranging between 0.02 and 0.06 when the idler-mode frequency $\omega_{i}$ is fixed at $2 \pi c / 1.596 \mu \mathrm{m}$. For the case of Fig. 3a), we assume a core radius $r=2 \mu \mathrm{m}$ and that the pump propagates in the $L P_{03}$ mode, whereas for Fig. 3c) $r=5 \mu \mathrm{m}$ and we assume that pump propagates in the $L P_{05}$ transverse mode. Note that in these plots, frequencies $\omega_{r}$ and $\omega_{s}$ have been expressed in terms of the frequency detunings $\Delta_{r}=\omega_{r}-\left(\omega_{p}-\omega_{i}\right) / 2$ and $\Delta_{s}=\omega_{s}-\left(\omega_{p}-\omega_{i}\right) / 2$, which satisfy the relation $\Delta_{r}=-\Delta_{s}=\Delta$ because of the energy
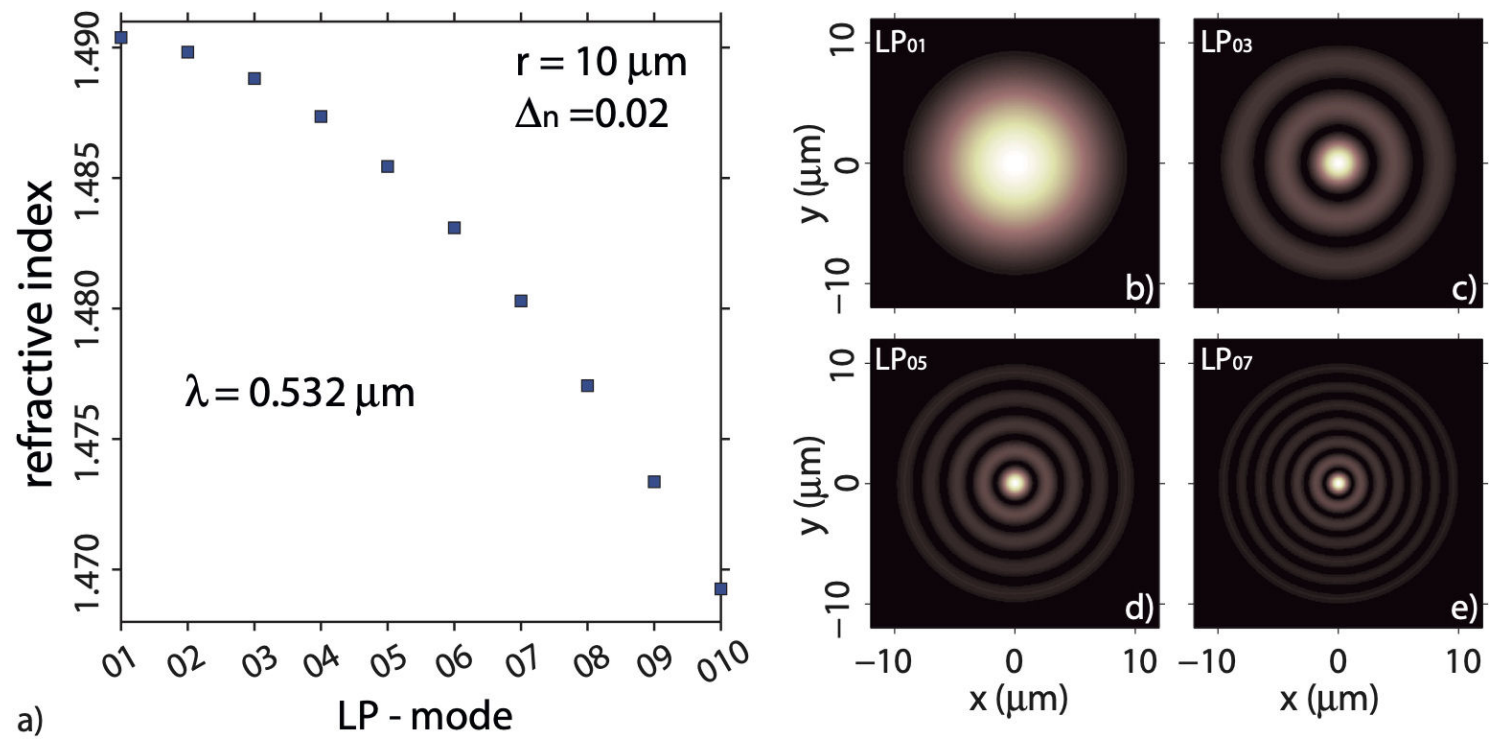

FIGURE 1. a) Effective refractive index of the first ten $\mathrm{LP}_{0 m}$ modes that can propagate along with a SIF with $r=10 \mu \mathrm{m}$ and $\Delta_{n}=0.02$. b)-e) Transverse intensity patterns of some of the modes supported by the fiber at $\lambda=0.532 \mu \mathrm{m}$. 

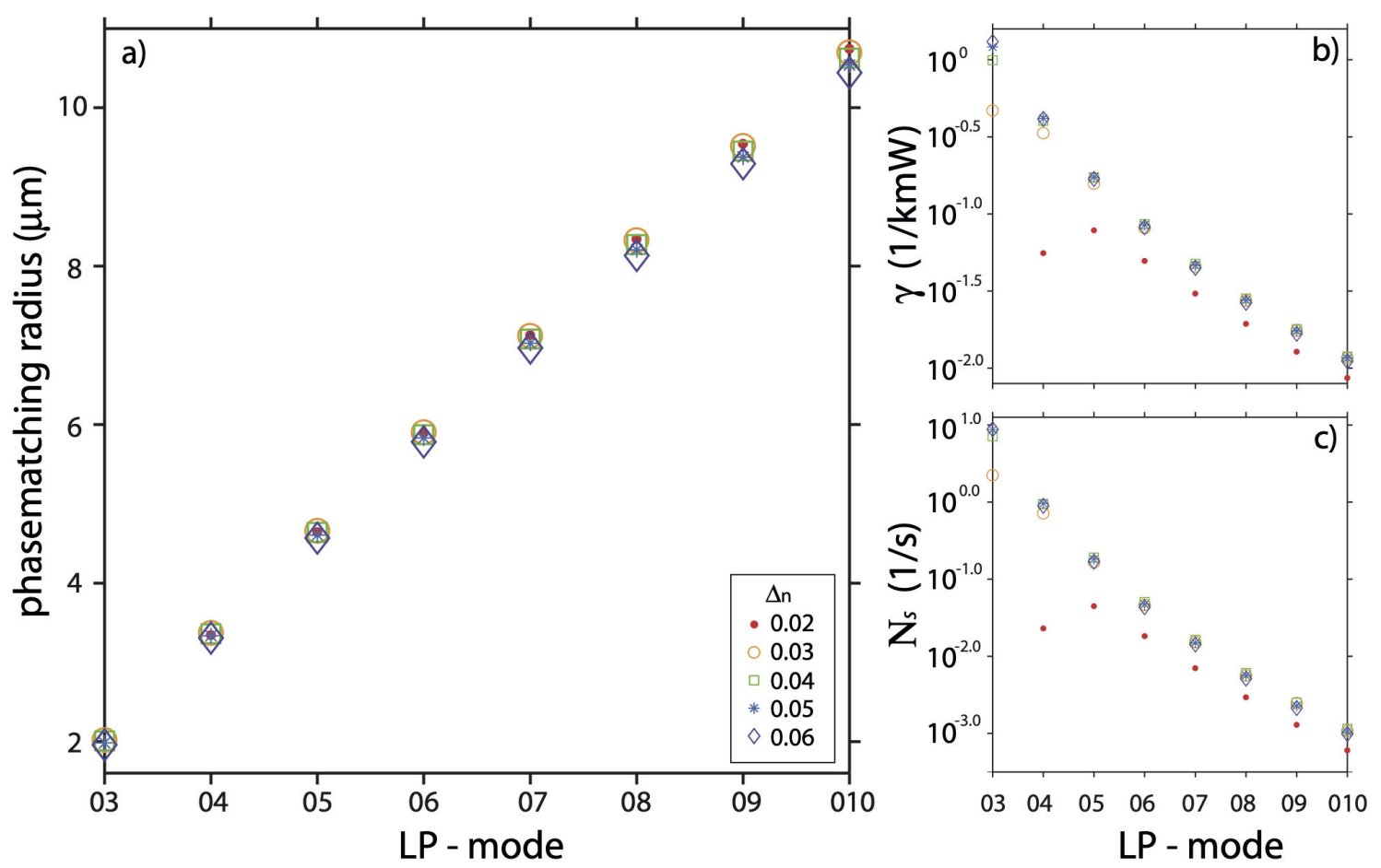

FIGURE 2. Phasematching for degenerate TOPDC at $1.596 \mu \mathrm{m}$ in multimode optical fibers. a) Phasematching radius $r_{\text {deg }}$ as function of the transverse mode order and the dielectric contrast. b) Nonlinear coefficient $\gamma$ corresponding to cases in panel a), see Eq. (6). c) TOSPDC emitted flux corresponding to a), see Eq. (5).

conservation constraint. In each case, the pump spatial mode corresponds to the lowest-order higher mode for which phase matching occurs within the considered $\Delta_{n}$ range.

From these figures, it can be appreciated that for each value of $r$ there is a continuum of dielectric contrasts for which phase matching occurs. However, note that for the case of $r=2 \mu \mathrm{m}$, there is no phase matching for $\Delta_{n}=0.02$. In fact, as the core radius decreases the phasematching is extinguished for small values of $\Delta_{n}$ [see Fig. 2a)]. Likewise, it can be seen from Figs. 3a) and Fig. 3c) that the values of $\Delta_{r}$ and $\Delta_{s}$ shrinks for larger radii, for which TOSPDC becomes feasible for pump fields propagating in fiber modes of a higher order. From this particular example, there is a wide range of configurations in which non-degenerate photon triplets can be generated in various spectral lines and with a pump that can be tuned at frequencies lying at the visible band.

\subsection{TOSPDC emitted flux}

Besides the phasematching required in TOSPDC, another relevant factor to consider is the source brightness [see Eq. (5)]. As already mentioned, in general terms, the emitted flux is proportional to the product $L p \gamma^{2}$. The quadratic dependence of $N_{s}$ on $\gamma$ can be exploited in favor of a high emission flux by considering fiber geometries in which $\gamma$ can be maximized. High $\gamma$ values are possible in fibers made from highly nonlinear materials such as bismuth and chalcogenides, which have a high nonlinear refractive index $[22,23]$, or in silica-based fibers with small core radii in which the spatial overlap among the four fields interacting along the fiber length can be optimized [see Eq. (6)]. It has been shown that for a specific $\Delta_{n}$, there is an optimal core radius for which the nonlinear coefficient reaches its highest value [29]. In Fig. 2b), we plot $\gamma$ [numerically calculated from Eq. (6)] for each phasematching radius obtained in Fig. 2a). We see that higher $\gamma$ values are attained for higher dielectric contrasts, at which phasematching is fulfilled for small radii by pumping with lower transverse modes. Lower modes as the $L P_{03}$ have better overlap with the fundamental mode. Even higher modes and small dielectric contrasts generally lead to small $\gamma$ 's; there are several configurations from which it is possible to get a reasonable TOSPDC emitted flux as can be seen in Fig. 2c). In this figure, we show the emitted flux $N_{s}$, calculated from Eq. (5), that corresponds to each core radius in panel (a), assuming a fiber length $L=5 \mathrm{~m}$, a pump average power $p=500 \mathrm{~mW}$, and a pulse duration of 10 ps.

Figure 2c) shows that because all other experimental parameters are fixed, the TOSPDC flux is determined by the nonlinear coefficient, fiber geometries with a high $\gamma$ value exhibit high values of $N_{s}$. Nevertheless, once the fiber parameters have been determined and set $\gamma$, the emitted flux can be increased, employing a longer fiber or a higher pump power. As we emphasized previously, photon triplet sources' successful implementations based on our proposed scheme rely on the feasibility of launching the pump field in a higherorder mode into an optical fiber. It is known that the amount 

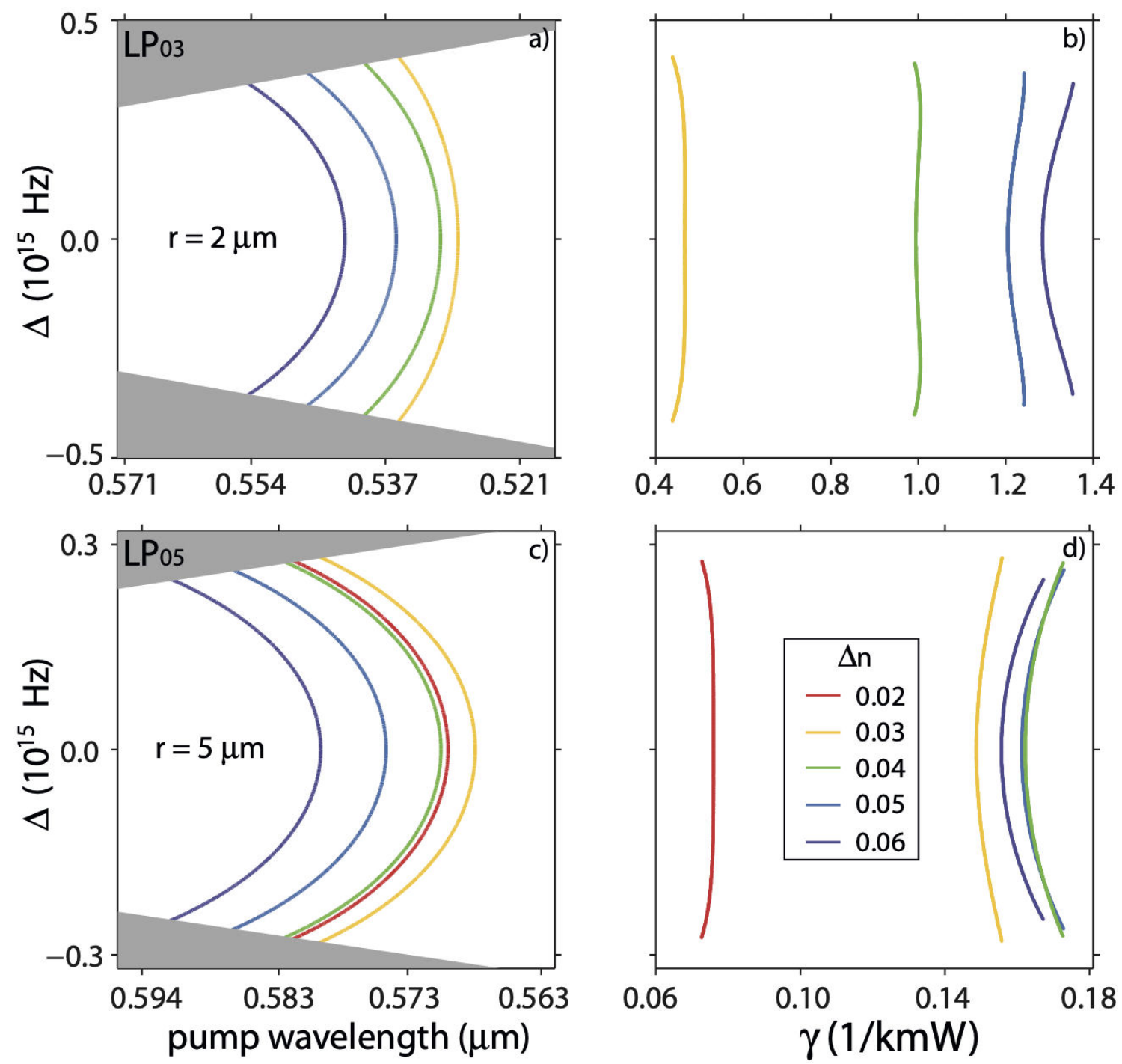

FIGURE 3. Phase-matched emission frequencies plotted as a function of pump frequency for different dielectric contrasts, assuming $\lambda_{i}=1.596 \mu \mathrm{m}$ and the following core radii and pump spatial modes: a) $r=2 \mu \mathrm{m}$ and $L P_{03}$, c) $r=5 \mu \mathrm{m}$ and $L P_{05}$. Panels b) and d) show the nonlinear coefficient $\gamma$ evaluated along the $\Delta k=0$ contours in a) and c), respectively.

of pump power that propagates in a given fiber transverse mode depends on the spatial overlap between the transverse intensity distribution of the laser beam and the corresponding mode. Thus, in conventional fibers, most of the pump power tends to be coupled to the fundamental mode since, in general, laser systems emit beams with approximately gaussian profiles. However, various experimental techniques have been proposed and implemented to excite selectively and controllably a particular higher-order mode in an optical fiber. In this way, it becomes possible to launch a significant amount of the pump power into the fiber, which can propagate in a higher-order mode. Some of the proposed schemes for excite higher-order modes are based on optical phase shifter [18], long-period gratings [19], hologram generated by spatial modulators [21], and PCF transitions [20]. Considering the advances in these and other techniques to excite higher-order fiber modes and the progress in the manufac- ture of fibers with engineered dispersion properties, we consider that the implementation of bright photon-triplet sources based on TOSPDC in optical fiber is an achievable goal.

Regarding the case of non-degenerate TOSPDC, we have evaluated numerically, from Eq. (6), the nonlinear coefficient $\gamma$ along the $\Delta k=0$ contours illustrated in Figs. 3a) and Fig. 3c). These results are shown in panels (b) and (d) of the same figure, where we can see that in general, as $\Delta_{n}$ decreases, the $\gamma$-values decrease. Likewise, it is possible to observe the usual trend that high values of the nonlinear coefficient are related to small core radii, which in the case of the four-field interaction we are describing is associated with a better spatial overlap between the pump transverse mode and the fundamental mode in which emitted photons propagate. It is noticeable that the $\gamma$-values obtained for the assumed fiber geometries are low compared with those that are possible to reach in PCFs or in nano-fibers used to exploit nonlinear ef- 

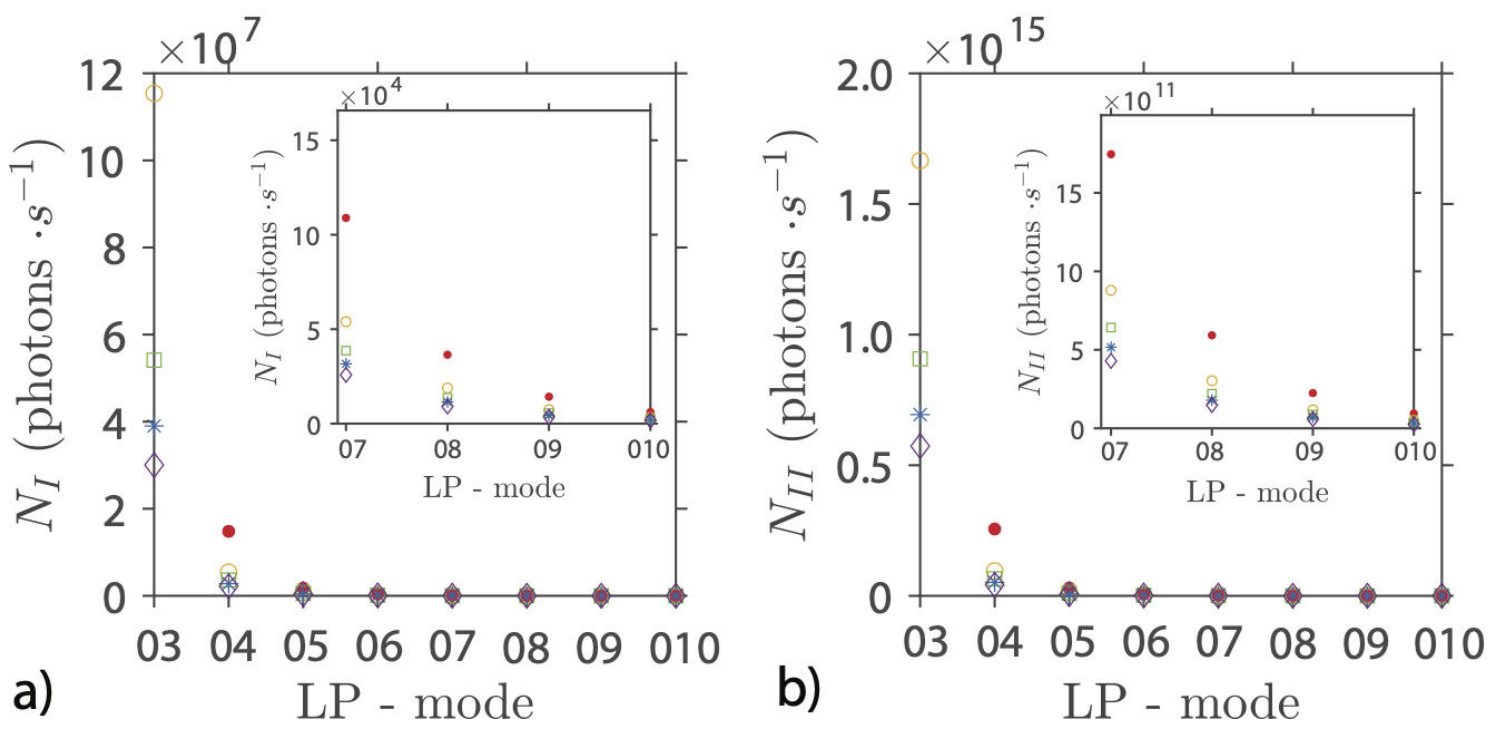

FIGURE 4. Seeded throughput from single a) and double seed b) contributions $N_{I}$ and $N_{I I}$, respectively. For a pulsed seed centered at $1596 \mathrm{~nm}$. Marker notation corresponds to that in Fig. 2.

fects. However, since what matters for obtaining a high photon-triplet flux is the value of the product $L p \gamma^{2}$, small $\gamma$ 's can be compensated with a longer fiber length and higher pump powers or both.

\subsection{Specific examples}

This section lets us consider some specific designs of photontriplet sources with degenerate emission frequencies at $1.596 \mu \mathrm{m}$, based on step-index fibers. Firstly, we present results for the spontaneous emission regime, for which a case was assumed a refractive index contrast $\Delta_{n}=0.04$. We consider a pump propagating in the $L P_{03}$ mode, for which the phasematching radius is $r_{d e g}=2.01 \mu \mathrm{m}$. For this configuration we obtain a nonlinear coefficient $\gamma=0.99(\mathrm{kmW})^{-1}$. Thus, by considering a fiber length $L=5 \mathrm{~m}$, a pump average power $p=500 \mathrm{~mW}$, and a pulse duration of $300 \mathrm{ps}$ the emission flux calculated from Eq. (5) is the 11.11 triplets per second. Now, if instead of the $L P_{03}$ mode, we assume that the pump propagates in the $L P_{05}$ mode while maintaining the same $\Delta_{n}$, the phasematching radius is $r_{d e g}=4.64$ $\mu \mathrm{m}$, for which $\gamma=0.17(\mathrm{~km} \mathrm{~W})^{-1}$. Under these conditions and assuming the same source parameters as above, the emission rate is $N_{s}=0.31 \mathrm{~s}^{-1}$, which is lower than that obtained for the case of the lower-order pump mode. The emitted flux could be increased by considering a longer fiber length or a higher pump power in both cases. For example, if for the second proposed design, we assume $L=25 \mathrm{~m}$ and $p=1 \mathrm{~W}$, the emitted flux can increase up to 3.11 triplets per second. Note that the need for long fiber length and high pump power can be overcome by advances in material science and fiber manufacturing, such that they lead to an increase in the effective nonlinear coefficient of fibers, a goal not far from being achieved.
Now, let us present results concerning the stimulated emission regime, for which we assume the same configuration as for the spontaneous case. From equations $(10)$, it is possible to obtain the throughput of stimulated photons due to single and double spectral overlap of the seed with output triplet modes. In this case, we assume a fiber length of $50 \mathrm{~cm}$. The seed will be assumed a pulsed electromagnetic field with the same duration as the pump, an average power of $10 \mathrm{mw}$, and a repetition rate of $10 \mathrm{MHz}$, centered at $1596 \mathrm{~nm}$, for which occurs the maximal stimulation. In these conditions, the contributions from $N_{I}$ and $N_{I I}$ are shown in Fig. 4a) and Fig. 4b), respectively. The figure shows that the obtained stimulated photons for each combination $(\Delta n, \mathrm{LP}$ - mode) are large enough to be easily detected without the need for single-photon detection. The maximal value for $N_{I}$ is of $11.5 \times 10^{7} \mathrm{~s}^{-1}$ and for $N_{I I}$ is of $1.6 \times 10^{15} \mathrm{~s}^{-1}$, values that occur for $\Delta n=0.03$ and the $L P_{03}$ transverse mode. In a real situation, the obtained throughput would be lower after filtering out the seed field.

\section{Conclusion}

This article presents an analysis of the third-order parametric down-conversion process in multimode optical fiber in both the spontaneous and stimulated emission regime. It has been shown that phasematching is achieved for a wide range of configurations in which the pump field is allowed to propagate in a higher-order mode while generated photons travel in the fundamental mode of the fiber. The phase matching properties were explored in step-index fibers with dielectric contrasts ranging between 0.02 and 0.06 . We found that phase matching occurs for fibers with conventional core radii (greater than one micron) in this interval. This feature becomes an advantage of the described scheme due to the fea- 
sibility of using longer fiber length and higher pump power, leading to higher emission rates. We also present values of the nonlinear coefficient and the emitted flux obtained for all fiber geometries considered in the study and propose some specific designs that can be useful for future implementations of fiber-based photon triplet sources. Although we have limited our analysis to SIF in this work, note that this applies equally to photonic crystal fibers, for which higher nonlinear coefficients and thus higher emission rates are obtained. The proposal can also be applied to integrated waveguides based on third-order nonlinear materials with a high nonlinear refractive index, leading to brighter sources due to the reduced transversal dimensions achievable with current micro and nanofabrication techniques. Also note, in integrated photonics, waveguide higher-order transverse modes excitation becomes possible with high coupling efficiencies. We expect our study contributes to the development of tripartite photon states and multipartite and multidimensional entanglement.

\section{Acknowledgments}

This work was supported by CONACYT, México, Cátedras CONACyT Project 709/2018.
1. D. C. Burnham and D. L. Weinberg, Observation of simultaneity in parametric production of optical photon pairs, Phys. Rev. Lett. 25 (1970) 84, https://doi.org/10.1103/ PhysRevLett.25.84

2. A. Zeilinger, Experiment and the foundations of quantum physics, Rev. Mod. Phys. 71 (1999) S288, https://doi. org/10.1103/RevModPhys.71.S288.

3. P. Kok, W. J. Munro, K. Nemoto, T. C. Ralph, J. P. Dowling, and G. J. Milburn, Linear optical quantum computing with photonic qubits, Rev. Mod. Phys. 79 (2007) 135 https : //doi.org/10.1103/RevModPhys.79.135.

4. M. Fiorentino, P. L. Voss, J. E. Sharping and P. Kumar, Allfiber photon-pair source for quantum communications, IEEE Photon. Technol. Lett. 14 (2002) 983, https://doi.org/ $10.1109 /$ LPT . 2002.1012406.

5. W. P. Grice, A. B. U'Ren and I. A. Walmsley, Eliminating frequency and space-time correlations in multiphoton states, Phys. Rev. A 64 (2001) 063815, https://doi.org/10.1103/ PhysRevA.64.063815.

6. K. Garay-Palmett, H. J. McGuinness, Offir Cohen, J. S. Lundeen, R. Rangel-Rojo, A. B. U'ren, M. G. Raymer, C. J. McKinstrie, S. Radic, and I. A. Walmsley, Photon pair-state preparation with tailored spectral properties by spontaneous four-wave mixing in photonic-crystal fiber, Opt. Express 15 (2007) 14870, https://doi.org/10.1364/OE.15.014870.

7. H. Hübel, D. R. Hamel, A. Fedrizzi, S. Ramelow, K. J. Resch and T. Jennewein, Direct generation of photon triplets using cascaded photon-pair sources, Nature 466 (2010) 601, https : //doi.org/10.1038/nature09175.

8. M. Corona, K. Garay-Palmett, and A. B. U'Ren, Experimental proposal for the generation of entangled photon triplets by third-order spontaneous parametric downconversion in optical fibers, Opt. Lett. 36 (2011) 190, https://doi.org/10. 1364 /OL.36.000190

9. K. Garay-Palmett, M. Corona, and A. B. U'Ren, Spontaneous parametric processes in optical fibers: a comparison, Rev. Mex. Fís. 57 (2011) 6.

10. C. Okoth, A. Cavanna, N. Y. Joly, and M. V. Chekhova, Seeded and unseeded high-order parametric down-conversion, Phys. Rev. A textbf99 (2019) 043809, https://doi.org/10. 1103/PhysRevA.99.043809.
11. A. Dot, A. Borne, B. Boulanger, K. Bencheikh, and J. A. Levenson, Quantum theory analysis of triple photons generated by a $\chi^{(3)}$ process, Phys. Rev. A 85 (2012) 023809, https: //doi.org/10.1103/PhysRevA.85.023809.

12. F. Gravier and B. Boulanger, Triple-photon generation: comparison between theory and experiment, J. Opt. Soc. Am. B 25 (2008) 98, https://doi.org/10.1364/JOSAB. 25.000098 .

13. J. Douady and B. Boulanger, Experimental demonstration of a pure third-order optical parametric downconversion process, Opt. Lett. 29 (2004) 2794, https : / / doi .org/10.1364/ OL.29.002794.

14. F. Domínguez-Serna, A. B. U'Ren, and K. Garay-Palmett, Third-order parametric down-conversion: A stimulated approach, Phys. Rev. A 101 (2020) 033813, https://doi. org/10.1103/PhysRevA.101.033813.

15. A. Cavanna, J. Hammer, C. Okoth, E. Ortiz-Ricardo, H. Cruz-Ramírez, K. Garay-Palmett, A. B. URen, M. H. Frosz, X. Jiang, N. Joly, and M. V. Chekhova, Progress toward third-order parametric-downconversion in optical fibers, Phys. Rev. A 101 (2020) 033840, https: / / doi.org/10.1103/ PhysRevA.101.033840.

16. S. Richard, K. Bencheikh, B. Boulanger, and J. A. Levenson, Semiclassical model of triple photons generation in optical fibers, Opt. Lett. 36 (2011) 3000, https : / / doi.org/10. $1364 /$ OL.36.003000.

17. M. Corona, K. Garay-Palmett, and A. B. U'Ren, Thirdorder spontaneous parametric down-conversion in thin optical fibers as a photon-triplet source, Phys. Rev A 84 (2011) 033823, https://doi.org/10.1103/PhysRevA.84. 033823 .

18. W. Q. Thornburg, B. J. Corrado, and X. D. Zhu, Selective launching of higher-order modes into an optical fiber with an optical phase shifter, Opt. Lett. 19 (1994) 454, https: //doi.org/10.1364/OL.19.000454.

19. S. Ramachandran, S. Ghalmi, J. W. Nicholson, M. F. Yan, P. Wisk, E. Monberg, and F. V. Dimarcello, Anomalous dispersion in a solid, silica-based fiber, Opt. Lett. 31 (2006) 2532, https://doi.org/10.1364/OL.31.002532. 
20. K. Lai, S. G. Leon-Saval, A. Witkowska, W. J. Wadsworth, and T. A. Birks, Wavelength-independent all-fiber mode converters, Opt. Lett. 32 (2007) 328, https://doi.org/10.1364/ OL.32.000328.

21. T. G. Euser, G. Whyte, M. Scharrer, J. S. Y. Chen, A. Abdolvand, J. Nold, C. F. Kaminski, and P. S. J. Russell, Dynamic control of higher-order modes in hollow-core photonic crystal fibers, Opt. Express 16 (2008) 17972, https://doi.org/ $10.1364 / \mathrm{OE} .16 .017972$.

22. J. M. Harbold, F. 'O. Ilday, F. W. Wise, J. S. Sanghera, V. Q. Nguyen, L. B. Shaw, and I. D. Aggarwal, Highly nonlinear AsS-Se glasses for all-optical switching, Opt. Lett. 27 (2002) 119, https://doi.org/10.1364/OL.27.000119.

23. H. Ebendorff-Heidepriem, P. Petropoulos, S. Asimakis, V. Finazzi, R. Moore, K. Frampton, F. Koizumi, D. Richardson, and T. Monro, Bismuth glass holey fibers with high nonlinearity, Opt. Express 12 (2004) 5082, https://doi.org/10. 1364 /OPEX.12.005082.

24. G. P. Agrawal, Nonlinear Fiber Optics, 4th Ed. (Elsevier, 2007).

25. M. Liscidini and J. E. Sipe, Stimulated emission tomography, Phys. Rev. Lett. 111 (2013) 193602, https://doi.org/ $10.1103 /$ PhysRevLett.111.193602.
26. B. Fang, O. Cohen, M. Liscidini, J. E. Sipe, and V. O. Lorenz, Fast and highly resolved capture of the joint spectral density of photon pairs, Optica 1 (2014) 281, https://doi.org/ $10.1364 /$ OP TICA.1.000281.

27. B. Fang, M. Liscidini, J. E. Sipe, and V. O. Lorenz, Multidimensional characterization of an entangled photon-pair source via stimulated emission tomography, Opt. Express 24 (2016) 10013, https://doi.org/10.1364/OE.24.010013.

28. V. Grubsky and J. Feinberg, Phase-matched third-harmonic UV generation using low-order modes in a glass micro-fiber, Opt. Commun. 274 (2007) 447 https://doi.org/10.1016/ j.optcom.2007.02.023.

29. M. Foster, K. Moll, and Alexander Gaeta, Optimal waveguide dimensions for nonlinear interactions, Opt. Express 12 (2004) 2880 https://doi.org/10.1364/OPEX.12. 002880 .

30. S. Leon-Saval, T. Birks, W. Wadsworth, P. St. J. Russell, and M. Mason, Supercontinuum generation in submicron fibre waveguides, Opt. Express 12 (2004) 2864, https://doi.org/ 10.1364 /OPEX.12.002864. 